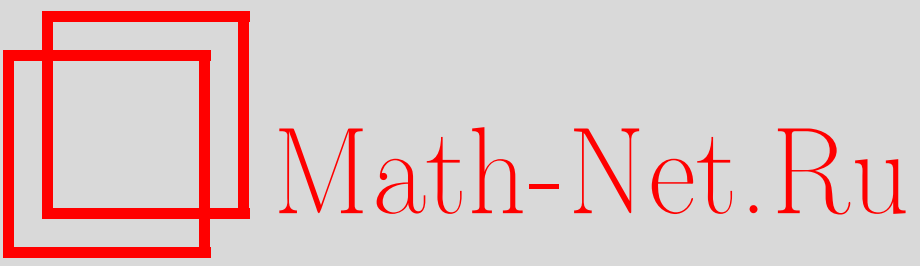

А. А. Туганбаев, Наследственные $\pi$-инъективные кольца, УМН, 1997, том 52 , выпуск 2, 187-188

DOI: https://doi.org/10.4213/rm844

Использование Общероссийского математического портала Math-Net.Ru подразумевает, что вы прочитали и согласны с пользовательским соглашением

http://www.mathnet.ru/rus/agreement

Параметры загрузки:

IP : 44.207 .124 .84

26 апреля 2023 г., $17: 57: 11$ 


\section{НАСЛЕДСТВЕННЫЕ $\pi$-ИНЪЕКТИВНЫЕ КОЛЬЦА}

\section{А. А. ТУГАНБАЕВ}

Все колца предполагаются ассоциативными и с ненулевой единицей. Через $E(M)$ обозначается инъективная оболочка модуля $M$. Модуль $M$ называется $\pi$-инъективным при выполнении следующих эквивалентных [1] условий: (1) для любых подмодулей $N_{1}, N_{2}$ модуля $M$ таких, что $N_{1} \cap N_{2}=0$, существует такое прямое разложение $M=M_{1} \oplus M_{2}$, что $N_{i} \subseteq M_{i} ;(2) f(M) \subseteq M$ для любого идемпотентного эндоморфизма $f$ модуля $E(M)$. Модуль $M$ называется непрерывным, если каждый его подмодуль является существенньм подмодулем прямого слагаемого модуля $M$, причем каждый подмодуль модуля $M$, изоморфньй прямому слагаемому модуля $M$, является прямым слагаемым модуля $M$. Все инъективные модули непрерывны. Непрерьвные модули $\pi$-инъективны [1]. Кольцо целых чисел является наследственным $\pi$-инъективньм, но не непрерывным кольцом. В [2] доказана

ТеОРема 1 [2]. Наследственное справа самоиндективное справа кольцо является полупростым артиновым кольцом.

Основным резултатом данной работы является

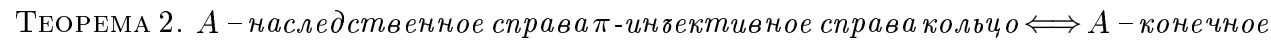
прямое произведение простых артиновых колец и наследственных справа нётеровых справа областей.

Доказательство теоремы 2 разобъем на ряд лемм. Через $Q_{\max }(A)$ обозначается максимальное правое кольцо частных колцца $A$. Модул, не содержащий бесконечных прямых сумм ненулевых подмодулей, называется конечномерным.

Лемма 1. Пусть $M-\pi$-инбективный модуль. Тогда:

(1) если $\operatorname{End}(M)$ не содержит бесконечного ортогонального множества идемпотентов, то $M-$ конечномерный модуль;

(2) если $Q$-существенное расширение модуля $M, f \in \operatorname{End}(Q), f^{2}=f$, mo $f(M) \subseteq M$.

ДоказАтЕЛЬСтво. (1) проверяется непосредственно. (2) Пусть $E=E(Q)$. Тогда $E=$ $E(M)=E_{1} \oplus E_{2}$, где $E_{1}=E(f(Q)), E_{2}=E((1-f)(Q))$. Пусть $g: E \rightarrow E_{1}-$ проекция с ядром $E_{2}$. По условию $f(M)=g(M) \subseteq M$.

Лемма 2. Пусть $A-\kappa о л ь ц о, ~ Q \equiv Q \max (A)$. Тогда:

(1) если $B$ - ненулевой правый идеал кольца $A$, то $B Q$ - ненулевой правый идеал кольча $Q, A \cap B Q$ - ненулевой правый идеал кольца $A$, причем $(B Q)_{A} u A \cap B Q-$ существенные расширения модуля $B_{A}$;

(2) если $B$ - замкнутый правый идеал кольца $A$, то $B=A \cap B Q$;

(3) если $B, C$ - такие правые идеаль кольца $A$, что $B \cap C=0$, то $B Q \cap C Q=0$;

(4) если $B_{A}=\bigoplus_{i \in I} B_{i}-$ правьй идеал кольца $A$, mо $(B Q)_{Q}=\bigoplus_{i \in I}\left(B_{i} Q\right)$;

(5) если $A$ - т-индективное справа кольцо, то $A$ содержит все идемпотенты кольца $Q$.

ДоказАтельство. (1) Достаточно доказать, что $(B Q)_{A}$ - существенное расширение модуля $B_{A}$. Пусть $0 \neq x=\sum_{i=1}^{n} b_{i} q_{i} \in B Q, b_{i} \in B, q_{i} \in Q$. Так как $Q_{A}$ - рациональное расширение модуля $A_{A}$, то существует такой элемент $a_{1} \in A$, что $0 \neq x a_{1}=\sum_{i=1}^{n} b_{i} q_{i} a_{1} \in B Q$, $q_{1} a_{1} \in A, b_{1} q_{1} a_{1} \in B$. Аналогично, существует такой элемент $a_{2} \in A$, что $0 \neq x a_{1} a_{2}=$ $\sum_{i=1}^{n} b_{i} q_{i} a_{1} a_{2} \in B Q, q_{2} a_{1} a_{2} \in A$. Кроме того, $b_{1} q_{1} a_{1} a_{2} \in B$. Поэтому существуют такие элементы $a_{1}, \ldots, a_{n} \in A$, что $0 \neq x a_{1} \cdots a_{n} \in B$. (2) следует из (1). (3) Пусть $\bar{B}, \bar{C}$ - замыкания правых идеалов $B$ и $C$ в кольце $A$. Тогда $\bar{B} \cap \bar{C}=0$. По (2) $\bar{B}=A \cap \bar{B} Q, \bar{C}=A \cap \bar{C} Q$. Тогда $0=(A \cap \bar{B} Q) \cap(A \cap \bar{C} Q)=A \cap(\bar{B} Q \cap \bar{C} Q)$. Так как $Q_{A}$ - существенное расширение модуля $A_{A}$, то $\bar{B} Q \cap \bar{C} Q=0$. Поэтому $B Q \cap C Q=0$. (4) следует из (3) и того, что $\left(\sum_{i \in J} B_{i}\right) Q=\sum_{i \in J}\left(B_{i} Q\right)$ для любого подмножества $J \subseteq I$. (5) следует из леммы $1(2)$ и того, что $Q_{A}$ - существенное расширение модуля $A_{A}$. 
Лемма 3. Пусть $A$-несингулярное справа кольцо, $Q \equiv Q \max (A)$. Тогда $Q-$ самоингективное справа регулярное кольчо, $Q=S \times T$, где $S$-строго регулярное самоиндективное кольцо, T порождается (как кольцо) идемпотентами.

ДоказАТЕЛЬСтво. См. [3, 2.31, 2.34, 2.35, 2.37].

ЛЕмма 4.

Пусть $A$ - несингулярное справа $\pi$-инвективное справа кольщо, $Q=Q_{\max }(A), S-$ правый идеал кольца $Q$. Тогда:

(1) $A=B \times T$, где $Q \max (B) \equiv S$ - строго регулярное самоингективное кольцо, $B$ редуцированное кольцо, содержащее все идемпотенты кольца $S, T$ - самоинвективное справ а регулярное кольчо, порожденное (как кольцо) идемпотентами;

(2) $S=(S \cap A) Q$;

(3) еслиt-кардинальное число, причем $S \cap A-t$-порожденныйправый идеалкольца $A$, то $S-t$-порожденный правый идеал кольца $A$;

(4) если $(S \cap A)_{A}=\bigoplus_{i \in I} A_{i}, \operatorname{mo~}_{Q}=\bigoplus_{i \in I} A_{i} Q$;

(5) если $S \cap A$ является прямой суммой счетно порожденных правых идеалов кольца $A$, то $S$ - проективный правый идеал кольча $Q$;

(6) если $S \cap A-$ проективныци правый идеал кольца $A$, то $S$ - проективный правый идеал кольца $Q$.

ДокАЗАТЕльство. (1) следует из лемм 3 и 2(5). (2) По лемме $3 Q$ - регулярное кольцо. Поэтому существует такое множество $\left\{e_{i}\right\}$ идемпотентов кольца $Q$, что $S=\sum_{i \in I} e_{i} Q$. По лемме $2(5)$ все идемпотенты $e_{i}$ лежат в $S \cap A$. Поэтому $S=(S \cap A) Q$. (3) следует из (2). (4) следует из (2) и леммы 2(4). (5) По (3) и (4) $S=\bigoplus_{i \in I} S_{i}$, где все $S_{i}$ - счетно порожденные правые идеалы регулярного колшца $Q$. Тогда все правые идеалы $S_{i}$ проективны $[4,2.15]$. Поэтому $S_{A}$ - проективньй модуль. (6) следует из (5) и того, что по теореме Капланского каждый проективньй модуль является прямой суммой счетно порожденных модулей.

Лемма 5. (1) $A-\pi$-инбективная справа область $\Longleftrightarrow A-$ конечномерная справа oбласть;

(2) $A$ - несингулярное справа т-ингективное справа кольцо, не содержащее бесконечного мно жества ортогональных идемпотентов $\Longleftrightarrow A-$ конечное прямое произведение простых артиновых колец и конечномерных справа областей.

ДокАЗАТЕЛЬСТво. Так как конечномерная справа область является правой областью Ope, то (1) проверяется непосредственно. (2) Импликация $\Longleftarrow$ проверяется с помощью (1). Импликация $\Longrightarrow$ следует из леммы 4(1).

ДокАЗАТЕЛЬСТво ТЕОРЕмЫ 2. Импликация $\Longleftarrow$ следует из леммы 5(2). Докажем импликацию $\Longrightarrow$. Пусть $Q \equiv Q_{\max }(A)$. Наследственное справа кольцо $A$ несингулярно справа. По лемме 4(6) $Q$ наследственно справа. По лемме $3 Q$ - самоинъективное справа кольцо. По теореме $1 Q$ - полупростое артиново кольо. Тогда $A$ конечномерно справа. Наследственное справа конечномерное справа кольц $A$ является нётеровым справа кольцом [3, следствие 5.20]. Далее применим лемму 5(2).

СлЕДСтвИЕ 1. Наследственное справа непрерывное справа кольчо является полупростым артиновым кольцом.

ДоКАЗАТЕЛЬСтво. Следует из теоремы 2 и того, что непрерывное справа несингулярное справа колшцо является регулярным (см., например, [1, теорема 7.1]).

\section{СПИСОК ЛИТЕРАТУРЫ}

[1] Jeremy L. // Canad. Math. Bull. 1974. V. 17. № 2. P. 217-228. [2] Osofsky B. L. // Proc. Amer. Math. Soc. 1968. V. 19. № 6. P. 1383-1384. [3] Goodearl K. R. Ring Theory. Nonsingular Ring and Modules. New York: Marcel Dekker, 1976. [4] Goodearl K. R. Von Neumann Regular Rings. London: Pitman, 1979. 